\title{
Imperatorin derivative OW1 inhibits the upregulation of TGF- $\beta$ and MMP-2 in renovascular hypertension-induced cardiac remodeling
}

\author{
NAN ZHOU ${ }^{1}$, YANING ZHU ${ }^{1}$, PENG ZHANG $^{1}$, YU ZHANG $^{2}$, \\ MINGYAO ZHOU ${ }^{3}$, TAO WANG ${ }^{2}$ and LANGCHONG $\mathrm{HE}^{2}$ \\ ${ }^{1}$ Department of Pharmacy, Shaanxi Provincial People's Hospital, Xi'an, Shaanxi 710068; \\ ${ }^{2}$ Institute of Material Medica, School of Pharmacy, Health Science Center, Xi'an Jiaotong University; \\ ${ }^{3}$ Xi'an Medical Emergency Center, Xi'an, Shaanxi 710061, P.R. China
}

Received October 22, 2014; Accepted January 26, 2016

DOI: $10.3892 /$ etm.2016.3172

\begin{abstract}
Chronic hypertension induces vascular and cardiac remodeling. OW1 is a novel imperatorin derivative that was previously reported to inhibit vascular remodeling and improve kidney function affected by hypertension. In the present study, the effect of OW1 on the cardiac remodeling induced by hypertension was investigated. OW1 inhibited vascular smooth muscle cell (VSMC) proliferation and the phenotypic modulation of VSMCs induced by angiotensin II (Ang II). The OW1-induced vasodilatation of rat cardiac arteries was evaluated in vitro. Renovascular hypertensive rats were developed using the two-kidney one-clip method and treated with OW1 (40 or $80 \mathrm{mg} / \mathrm{kg} /$ day) or nifedipine (30 mg/kg per day) for 5 weeks. OW1 markedly reduced the systolic and diastolic blood pressure compared with that in the hypertension group or the respective baseline value during the first week. OW1 also reduced cardiac weight, and the concentrations of Ang II, aldosterone and transforming growth factor- $\beta 1$ (TGF- $\beta 1$ ). Histological examination demonstrated that OW1 exerted an inhibitory effect on vascular and cardiac remodeling. These inhibitory effects were associated with decreased cardiac levels of Ang II, matrix metalloproteinase- 2 and TGF- $\beta_{1}$ in the hypertensive rats. In summary, OW1 exhibited a clear antihypertensive effect. More importantly, it inhibited vascular and cardiovascular remodeling, which may reduce the risk of hypertension-induced cardiovascular diseases. These results have potential implications in the development of new antihypertensive drugs.
\end{abstract}

Correspondence to: Professor Langchong He, Institute of Material Medica, School of Pharmacy, Health Science Center, Xi'an Jiaotong University, 76 Yanta West Street, Xi'an, Shaanxi 710061, P.R. China E-mail: helc@mail.xjtu.edu.cn

Key words: imperatorin derivative, OW1, blood pressure, two-kidney one-clip hypertensive rats, cardiac remodeling

\section{Introduction}

Chronic hypertension induces vascular and cardiac remodeling (1-4). Remodeling involves the hypertrophy of cells and the excessive deposition of extracellular matrix (ECM) within tissues, leading to arterial wall hypertrophy, abnormal myocardial stiffness and an impairment of left ventricular (LV) function $(2,5,6)$. A major component of the myocardial ECM is collagen (1). In hypertension, the key mediators of increases in the levels of collagen include angiotensin II (Ang II) and transforming growth factor- $\beta$ (TGF- $\beta$ ) $(1,7)$; the hypertrophic effects of Ang II are exerted via increased levels of reactive oxygen species (ROS) and TGF- $\beta$ (7-10), which promote matrix metalloproteinase (MMP) activation. Notably, the overexpression of MMP-2 induces severe ventricular remodeling and systolic dysfunction in the absence of superimposed injury, which may be due to MMP-2 activity affecting intracellular targets and potentially impairing myocardial contractility (11-13).

Numerous treatments for hypertension have been investigated, with a focus on reducing the risk of hypertension-induced cardiovascular diseases. In the present study, a novel imperatorin derivative, 2-(3,4-dimethoxyphenyl)-2-isopropyl-5-[(2-[(7-oxo7H-furo[3,2-g] chromen-4-yl)-oxy]ethyl)amino]pentanenitrile (OW1; Fig. 1), which previously exhibited hypotensive effects in two-kidney, one-clip (2K1C) renovascular hypertensive rats (14), was further investigated. In the previous study, OW1 decreased the generation of Ang II, inhibited vascular remodeling of the thoracic aorta and improved the kidney function affected by hypertension (14). In the $2 \mathrm{~K} 1 \mathrm{C}$ model of hypertension, activation of the renin-angiotensin-aldosterone system (RAAS) occurs, which leads to increased cardiac myocyte thickness and is associated with imbalanced MMP activity (15). The present study aimed to explore the effect of OW1 on the cardiac remodeling induced by hypertension.

\section{Materials and methods}

Experimental rats. A total of 55 male Sprague-Dawley (SD) rats (age, 6-8 weeks; body weight, 200-230 g) and two male SD rats (age, 3-4 weeks) were obtained from the Animal 
Center of Xi'an Jiaotong University (Xi'an, China). The rats were maintained at room temperature under a 12-h light/dark cycle with ad libitum access to tap water and standard rat feed. The rats were cared for in accordance with the principles of the National Institutes of Health Guide for the Care and Use of Laboratory Animals (16). All protocols were approved by the Institutional Animal Investigation Committee of Xi'an Jiaotong University.

Preparation of $O W 1$. OW1 was prepared in the Natural Drug Research and Engineering Center of Xi'an Jiaotong University using xanthotoxin (Meryer Chemical Technology Co., Ltd., Shanghai, China) as the starting material. OW1 was synthesized via a previously reported method comprising six reaction steps, with characterization of the chemical structures (17).

Measurement of the vascular effects of OWl in rat coronary artery (CA) rings. A total of 10 male SD rats aged 6-8 weeks were anesthetized by intraperitoneal injection with $10 \%$ chloral hydrate $(350 \mathrm{mg} / \mathrm{kg}$ body weight; Shanghai Jinjinle Industry Co., Ltd., Shanghai, China) and sacrificed by decapitation. The superior CAs $(2 \mathrm{~mm})$ were dissected free of fat and connective tissue and mounted in a Multi Wire Myograph System (Danish Myo Technology A/S, Inc., Skejbyparken, Denmark). The vessels were maintained at $37^{\circ} \mathrm{C}$ in physiological Krebs-Henseleit solution ( $\mathrm{pH}$ 7.4) through which a gaseous mixture of $95 \% \mathrm{O}_{2}$ and $5 \% \mathrm{CO}_{2}$ was bubbled. Following a $30 \mathrm{~min}$ equilibration period, the CA segments were equilibrated for $1.5 \mathrm{~h}$ with a resting tension of $3 \mathrm{mN}$ prior to the initiation of testing. The contractile capacity of each CA segment was tested by exposure to a $\mathrm{K}^{+}$-rich Krebs-Henseleit solution (containing $4.45 \mathrm{~g} / \mathrm{l} \mathrm{KCl}$ ), in which $\mathrm{NaCl}$ was exchanged for an equimolar concentration of $\mathrm{KCl}$. When two reproducible contractions were achieved the $\mathrm{CA}$ segments were further evaluated. Following equilibration, the segments were pre-contracted with $\mathrm{KCl}(60 \mathrm{mM})$. Once the sustained tension was obtained, OW1 $(0.1 \mu \mathrm{M}-0.1 \mathrm{mM})$ was added at increasing concentrations to the baths, and concentration-response curves were constructed.

Proliferation assay. The SD rats aged 3-4 weeks were sacrificed by decapitation and the thoracic aortas were removed. Vascular smooth muscle cells (VSMCs) were isolated from the thoracic aorta as previously described (18). The cells were grown at $37^{\circ} \mathrm{C}$ in a $95 \% \mathrm{O}_{2}$ and $5 \% \mathrm{CO}_{2}$ atmosphere in Dulbecco's modified Eagle's medium (Beijing Skywing Technology Co., Ltd., Beijing, China) containing 10\% fetal bovine serum, $2 \%$ penicillin $(100 \mathrm{U} / \mathrm{ml}$; North China Pharmaceutical Group Corporation, Shijiazhuang, China), and $1 \%$ L-glutamine. Cells at passages $4-8$ were used in experiments. The VSMCs were exposed to Ang II $(1 \mu \mathrm{M}$; Sigma-Aldrich, St. Louis, MO, USA) for $48 \mathrm{~h}$ in the presence or absence of OW1 $(5,10$ or $20 \mu \mathrm{M})$. Cell proliferation was analyzed by the methyl thiazolyl tetrazolium assay (MTT) method (19).

Induction of the 2 K1C model of hypertension. A total of 45 male SD rats aged 6-8 weeks were used to establish the $2 \mathrm{~K} 1 \mathrm{C}$ rat model of hypertension. Surgical procedures were

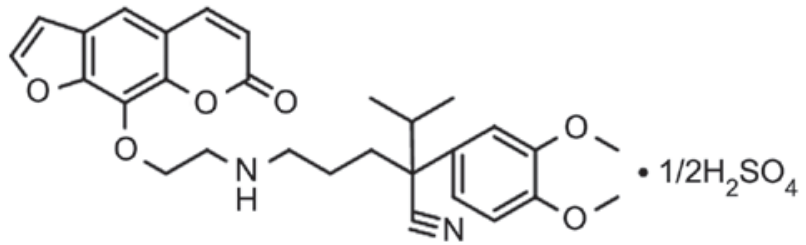

Figure 1. Chemical structure of OW1.

performed under $10 \%$ chloral hydrate anesthesia $(350 \mathrm{mg} / \mathrm{kg}$ body weight, intraperitoneally). $2 \mathrm{~K} 1 \mathrm{C}$ modeling was conducted by separating the left renal artery through an abdominal approach and placing it in a $0.3 \mathrm{~mm}$ silver clip. Rats in the sham-operated group underwent the same procedure, with the exception that the renal artery was not clipped. The rats were placed into single cages and their airways were kept open until they regained consciousness (20). After 3 days, the rats were injected once daily with penicillin $\mathrm{G}$ (8 million units; North China Pharmaceutical Group Co., Ltd., Shijiazhuang, China) for 5 days. After 4 weeks, blood pressure was measured by the tail-cuff method (21), using a Coda-Volume Pressure Recording (VPR) system (Kent Scientific Corporation, Torrington, CT, USA).

On week 6 after the surgery, rats with a systolic blood pressure $(\mathrm{SBP}) \geq 140 \mathrm{mmHg}$ were considered to be hypertensive. At the end of week 6, the SBP was very stable and rats with a SBP $>160 \mathrm{mmHg}$ were randomly divided into four groups: i) Hypertension group, comprising rats intragastrically treated with vehicle (ultrapure water); ii) nifedipine group (30 mg/kg/day); iii) OW1 high dose group (OW1-H; $80 \mathrm{mg} / \mathrm{kg} / \mathrm{day}$ ); iv) OW1 low dose group (OW1-L; $40 \mathrm{mg} / \mathrm{kg} /$ day). A sham-operated group was also established. Drugs or vehicle were administered by gavage once a day for 5 weeks. The blood pressure of the rats in each group was measured by the tail-cuff method.

Plasma samples. When the treatment period was complete, the rats were sacrificed with $20 \%$ urethane $(1 \mathrm{~g} / \mathrm{kg}$, intraperitoneally; Yangzhou Xinhua Chemical Industry Co., Ltd., Yangzhou, China) in the morning after overnight fasting, and blood samples were then collected in vacuum tubes with ethylenediamine tetraacetic acid as an anticoagulant. The blood samples were centrifuged at $1,000 \mathrm{x}$ g for $15 \mathrm{~min}$ at $4^{\circ} \mathrm{C}$ to separate the plasma, which was then frozen at $-80^{\circ} \mathrm{C}$ until required for testing. Levels of aldosterone (ALD) in plasma were detected using a radioimmunoassay kit (Beijing Sino-UK Institute of Biological Technology, Beijing, China). Levels of TGF- $\beta_{1}$ in the plasma were quantified using an enzyme-linked immunosorbent assay kit (ML-Elisa-0014; Shanghai Fuzhong Bio Tech Co., Ltd., Shanghai, China).

Harvesting of the hearts. When the treatment period was complete, the rats were anesthetized with urethane as described above. The thoracic cavity was opened to expose the heart, which continued to beat. The heart was rapidly removed, rinsed in ice-cold $0.9 \%$ saline solution, blotted and weighed. The two ventricles from the heart were isolated, cut into two fragments by a mid-ventricular coronal section and stored in phosphate-buffered $10 \%$ formalin $(\mathrm{pH} 7.3)$ for 

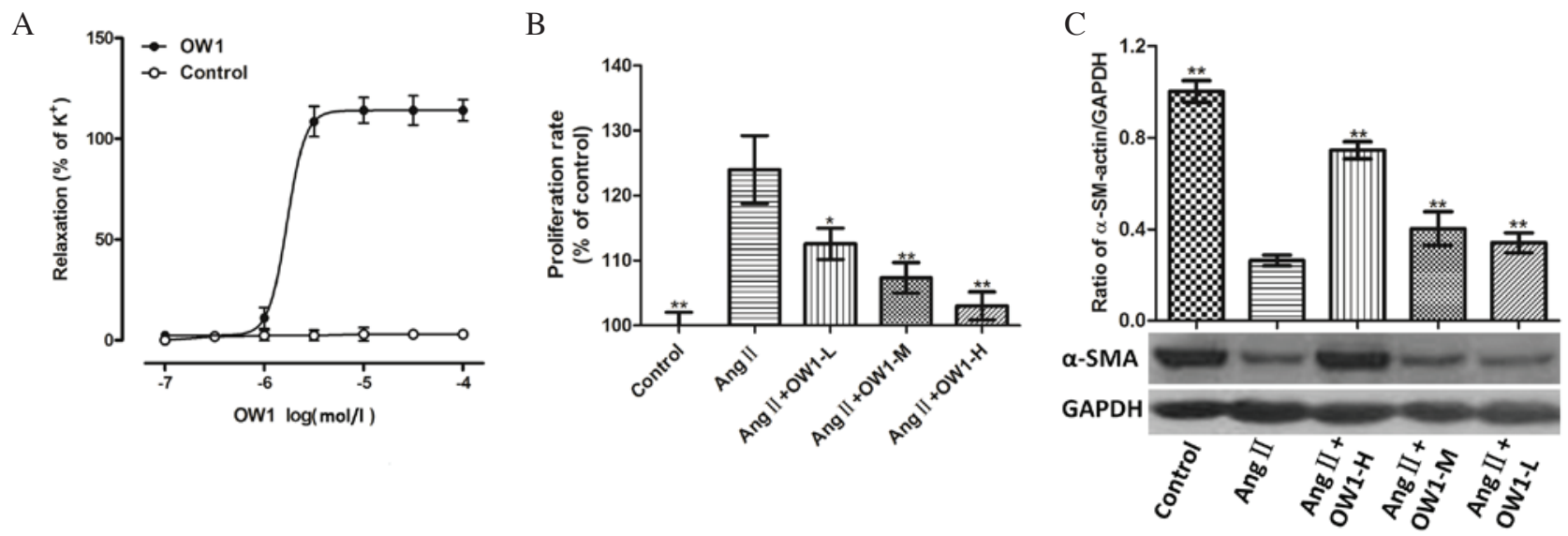

Figure 2.OW1 induced vasodilatation in rat cardiac arteries and had an inhibitory effect on Ang II-induced VSMC proliferation in vitro.(A) Concentration-response curve of OW1 for the vasodilatation effect on rat cardiac artery pre-contracted with $\mathrm{KCl}\left(\mathrm{K}^{+}\right)$. All values are means \pm standard error of the mean $(\mathrm{SEM}$; $\mathrm{n}=10)$. (B) MTT assay of VSMCs in the presence or absence of Ang II and OW1-L (5 $\mu \mathrm{M})$, OW1-M (10 $\mu \mathrm{M})$ and OW1-H (20 $\mu \mathrm{M})$. (C) Effect of OW1 on the expression of $\alpha$-SMA in VSMCs. Data represent normalized (against GAPDH) densitometric values (means \pm SEM, $n=3$ ). Representative blots are shown. ${ }^{*} \mathrm{P}<0.05$, ${ }^{* *} \mathrm{P}<0.01$ vs. Ang II. OW1, an imperatorin derivative; Ang-II, angiotensin II; VSMC, vascular smooth muscle cell; MTT, methyl thiazolyl tetrazolium; SMA, smooth muscle actin; GAPDH, glyceraldehyde-3-phosphate dehydrogenase.

histological examination. The remainder of the heart was frozen and stored at $-80^{\circ} \mathrm{C}$ until required for biochemical examination. The levels of Ang II in the heart were detected using a radioimmunoassay kit (Beijing Sino-UK Institute of Biological Technology).

Histology. The thoracic aorta and heart were stained with hematoxylin and eosin (H\&E) and Masson's trichrome respectively. Images were viewed and captured using the Leica Q550CW Image Analysis System (Leica Microsystems $\mathrm{GmbH}$, Wetzlar, Germany) and analyzed using ImagePro Plus version 3.0 (Media Cybernetics,. Rockville, MD, USA) to determine the aortic cross-sectional area (CSA) and media to lumen $(\mathrm{M} / \mathrm{L})$ ratio.

Western blot analysis. VSMCs exposed to Ang II in the presence or absence of OW1 $(5,10$ or $20 \mu \mathrm{M})$ as in the proliferation assay, or thoracic aortas from individual rats in each group were lysed in radioimmunoprecipitation assay lysis buffer containing $0.1 \mathrm{M}$ phenylmethylsulfonyl fluoride and centrifuged at $13,400 \mathrm{x} \mathrm{g}$ for $20 \mathrm{~min}$. The protein concentrations of the supernatants were determined by bicinchoninic acid (BCA) Protein Assay (Applygen Technologies, Inc., Beijing, China). Extracts were boiled in a 1:1 ratio with loading buffer containing Tris (125 mmol/1, pH 6.8), 4\% w/v sodium dodecylsulfate, $10 \% \mathrm{v} / \mathrm{v}$ glycerol, $4 \% \mathrm{v} / \mathrm{v} 2$-mercaptoethanol, and $2 \mathrm{mg} / \mathrm{ml}$ bromophenol blue. Equal amounts of protein (30-60 $\mu \mathrm{g}$ ) were resolved by sodium dodecyl sulfate-polyacrylamide gel electrophoresis (SDS-PAGE; 10\%) and transferred to nitrocellulose membranes (GE Healthcare Life Sciences, Logan, UT, USA). The membranes were incubated overnight at $4{ }^{\circ} \mathrm{C}$ with mouse anti- $\alpha$-smooth muscle actin polyclonal antibody ( $\alpha$-SMA; 1:200; BM0002; Wuhan Boster Biological Technology, Ltd., Wuhan, China), rabbit anti-MMP-2 polyclonal antibody (1:500; ab37150; Abcam, Hangzhou, China), rabbit anti-collagen I polyclonal antibody (1:100; bs-10423R; Beijing Biosynthesis Biotechnology Co., Ltd., Beijing, China), and rabbit anti-glyceraldehyde-3-phosphate dehydrogenase polyclonal antibody (GAPDH; 1:500; AB-P-R 00; Hangzhou
Xianzhi Bio Tech Co., Ltd., Hangzhou, China) as an internal control. After wshing the membranes three times with phosphate-buffered saline containing Tween-20, the membranes were incubated with horseradish peroxidase-conjugated secondary antibodies $(1: 2,000 ; 31460$; Shaanxi Xianfeng Biotechnology Co., Ltd., Shaanxi, China) for $45 \mathrm{~min}$ at $37^{\circ} \mathrm{C}$, followed by incubation with Western Blotting Luminol Reagent (Shanghai Tuoran Technology Co., Ltd., Shanghai, China). Images of the membranes were captured using an image acquisition and analysis system (ChampGel ${ }^{\mathrm{TM}}$ 6000; Beijing Sage Creation Science Co., Ltd., Beijing, China), and the relative expression levels were determined using Image J software, version 1.46r (National Institutes of Health, Bethesda, MA, USA).

Statistical analysis. Data are expressed as the mean \pm standard error of the mean. One-way analysis of variance followed by a Tukey's multiple comparison test was used to test the significance between three or more groups. $\mathrm{P}<0.05$ was considered to indicate a statistically significant difference.

\section{Results}

OW1 induces vasodilatation of rat coronary arteries and inhibits Ang II-induced VSMC proliferation in vitro. OW1 $(0.1 \mu \mathrm{M}-0.1 \mathrm{mM})$ relaxed the CA segments that were pre-contracted with $\mathrm{KCl}$ in a concentration-dependent manner. The maximum relaxation effect (Emax) of OW1 on the CA segments was $114.1 \pm 2.3 \%$ and the median effective concentration $\left(\mathrm{EC}_{50}\right.$ ) was $1.00 \pm 0.05 \mu \mathrm{M}$ (Fig. $2 \mathrm{~A}$ ).

The MTT assay showed that OW1 $(5 \mu \mathrm{M})$ inhibited Ang II-induced VSMC proliferation (Fig. 2B). Higher concentrations of OW1 (10 or $20 \mu \mathrm{M})$ exhibited a stronger inhibitory effect ( $\mathrm{P}<0.01$ vs. Ang II; Fig. 2B). The examination of the expression levels of $\alpha$-SMA by western blot analysis showed that the $\alpha$-SMA protein levels were lower in the VSMCs treated with Ang II alone. The OW1 treatment (5, 10 and $20 \mu \mathrm{M})$ attenuated the Ang-II-mediated inhibition of $\alpha$-SMA levels in a concentration-dependent manner (Fig. 2C). 

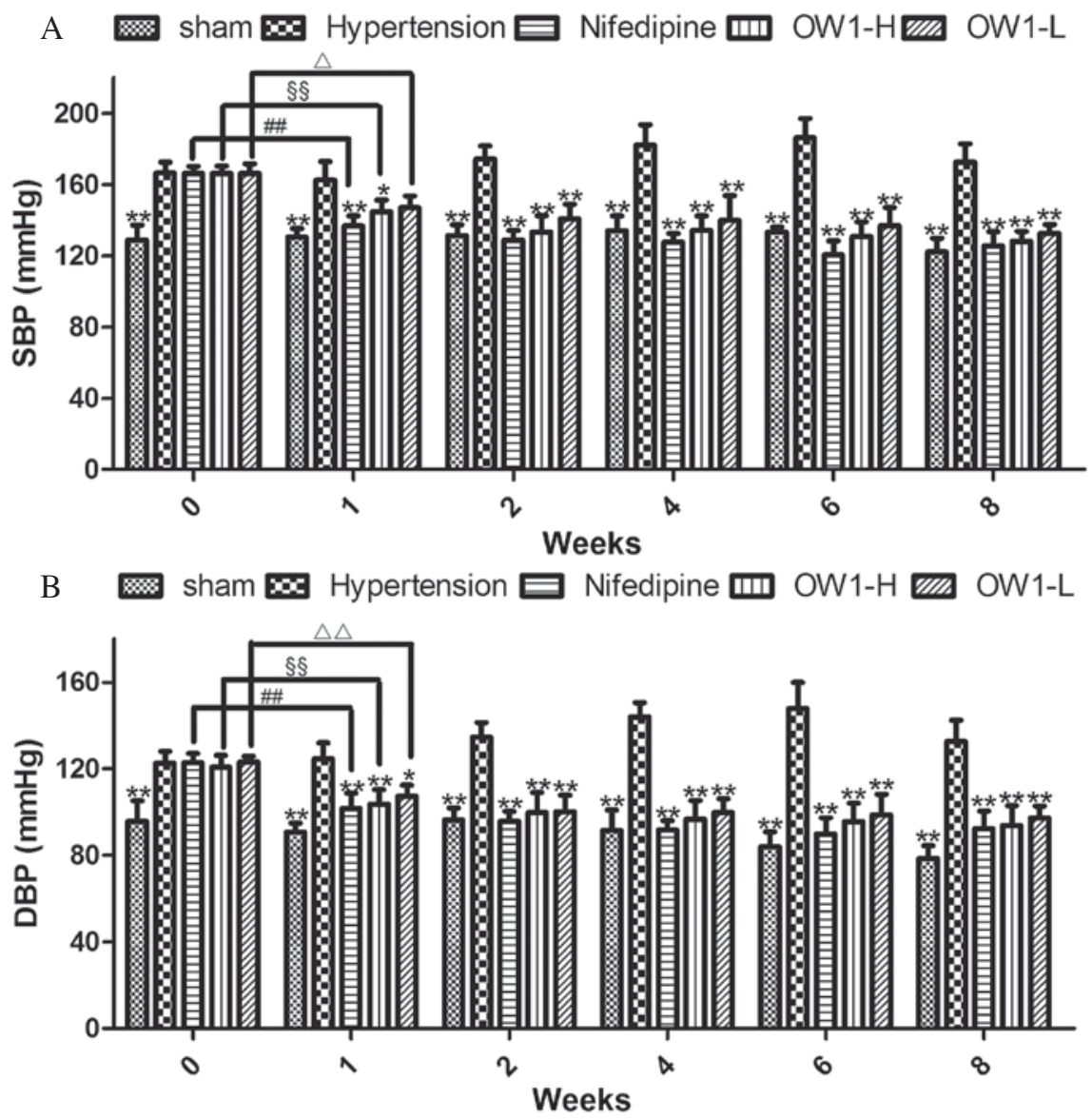

Figure 3. Effect of OW1 on blood pressure in $2 \mathrm{~K} 1 \mathrm{C}$ hypertensive rats. Effect of OW1-H ( $80 \mathrm{mg} / \mathrm{kg} / \mathrm{day})$ and OW1-L (40 mg/kg/day) on (A) systolic blood pressure (SBP) and (B) diastolic blood pressure (DBP). All values are means \pm standard error of the mean $(\mathrm{n}=6) .{ }^{*} \mathrm{P}<0.05$, ${ }^{* * *} \mathrm{P}<0.01$ vs. the hypertension group; ${ }^{\# \#} \mathrm{P}<0.01$ vs. the Nifedipine group; ${ }^{\$ \$} \mathrm{P}<0.01$ vs. the OW1-H group; ${ }^{\circ} \mathrm{P}<0.05$ vs. the OW1-L group and ${ }^{\Delta \Delta} \mathrm{P}<0.01$ vs. the OW1-L group. $2 \mathrm{~K} 1 \mathrm{C}$, two-kidney one-clip; OW1-H, OW1 high dose group; OW1-L, OW1 low dose group.

OW1 reduces blood pressure in $2 \mathrm{~K} 1 \mathrm{C}$-induced hypertension. The baseline blood pressure values among the groups were not observed to differ significantly, with the exception that the sham-operated group had a lower SBP and diastolic blood pressure (DBP) compared with the groups subjected to the 2K1C modeling procedure $(\mathrm{P}<0.01$; Fig. 3). During week 1 of treatment, the SBP and DBP were markedly reduced in the nifedipine, OW1-H and OW1-L groups compared with those in the hypertension group $(\mathrm{P}<0.05)$ or the baseline value of the respective group $(\mathrm{P}<0.05)$.

OWl reduces cardiac weight, and Ang II, ALD and TGF- $\beta 1$ concentration. The mean heart weight was higher in the hypertension group compared with the sham group $(\mathrm{P}<0.05)$. Compared with the hypertension group, the heart weight of the OW1-H group was significantly decreased ( $\mathrm{P}<0.05$; Fig. 4A). However, the mean heart weights in the nifedipine and OW1-L groups were not significantly different from that in the hypertension group. The cardiac concentration of Ang II was lower in the OW1 groups compared with the hypertension group $(\mathrm{P}<0.05$; Fig. 4B), but no difference was observed between the nifedipine and hypertension groups. Plasma levels of ALD and TGF- $\beta_{1}$ were elevated in the hypertension group ( $\mathrm{P}<0.01$ vs. sham group) and were reduced in the two OW1 groups but not in the nifedipine group $(\mathrm{P}<0.01$ vs. hypertension group; Fig. $4 \mathrm{C}$ and D).
OWl attenuates cardiac remodeling. Cardiac remodeling was observed in the hypertension groups, with significant myocardial fibrosis (Fig. 5A). In particular, the area around the microvasculature exhibited serious fibrosis (Fig. 5B). OW1 reduced the fibrosis to a greater extent than nifedipine, even though the nifedipine group had only a slight amount of myocardial fibrosis. Fibrosis in the myocardium or around the microvasculature was difficult to identify in the OW1 groups. Western blot analysis verified the above results. The expression of collagen $I$ in the heart was higher in the hypertension group compared with the sham group $(\mathrm{P}<0.01)$. The OW1 groups showed lower expression levels of collagen I compared with that in the hypertension group $(\mathrm{P}<0.01)$. However, the nifedipine group exhibited no difference from the hypertension group. The MMP-2 level was also elevated in the hypertension group ( $\mathrm{P}<0.01 \mathrm{vs}$. sham group). OW1-H and nifedipine reduced the MMP-2 level, but no reduction was observed in the OW1-L group (Fig. 5C and D).

OWl attenuates vascular remodeling. Serious arterial wall hypertrophy was observed in the hypertension group (Fig. 4). The vascular wall was thickened markedly with significant increases in VSMC numbers. The aortic CSA and $\mathrm{M} / \mathrm{L}$ ratio in the hypertension group were higher than those in the sham group $(\mathrm{P}<0.01)$. Correspondingly, reduced CSA and $\mathrm{M} / \mathrm{L}$ ratios were observed in the OW1 and nifedipine groups $(\mathrm{P}<0.01$ vs. hypertension group; Fig. 6). 
A

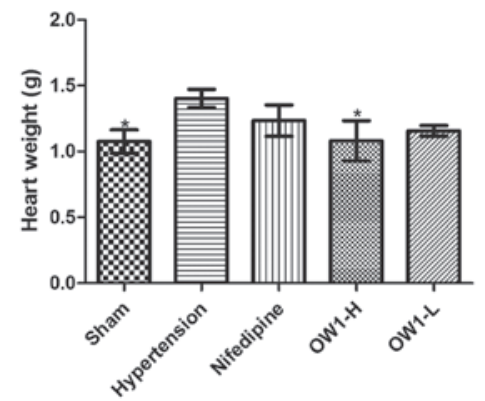

$\mathrm{C}$

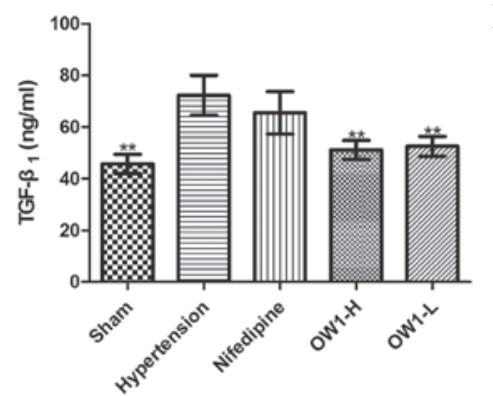

B

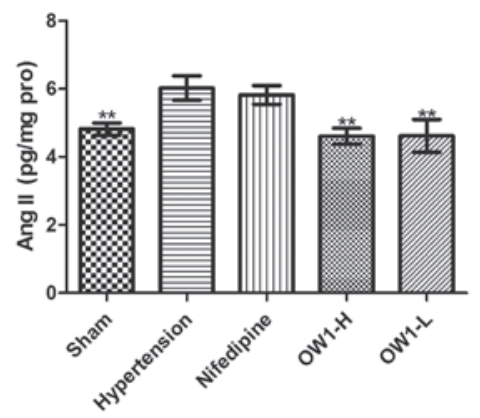

D

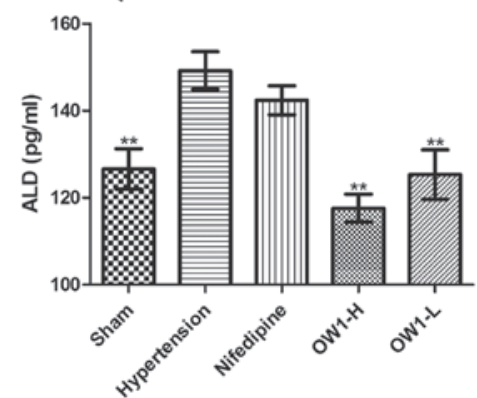

Figure 4. OW1 reduces cardiac weight, and Ang II, ALD and TGF- $\beta_{1}$ concentrations. Effect of OW1-H ( $\left.80 \mathrm{mg} / \mathrm{kg} / \mathrm{day}\right)$ and OW1-L (40 mg/kg/day) on (A) cardiac weights, (B) concentration of Ang II in cardiac tissue, and plasma levels of (C) TGF- $\beta_{1}$ and (D) ALD. All values are means \pm standard error of the mean $(\mathrm{n}=8) .{ }^{* *} \mathrm{P}<0.01,{ }^{*} \mathrm{P}<0.05$ vs. the hypertension group. OW1, an imperatorin derivative; Ang II, angiotensin II; ALD, aldosterone; TGF, transforming growth factor.
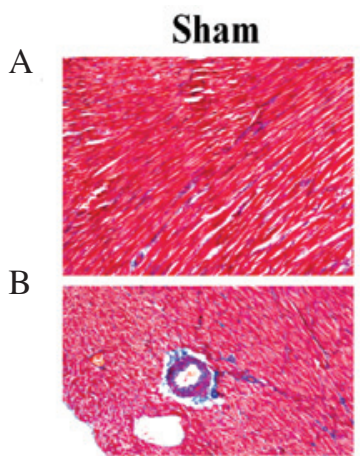

$\mathrm{C}$

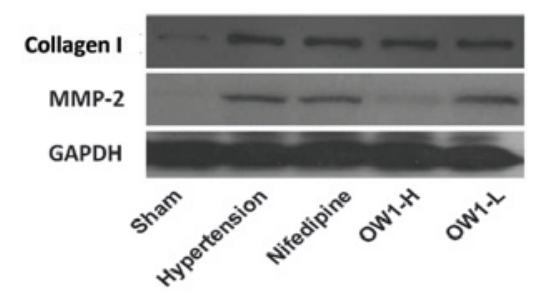

Nifedipine
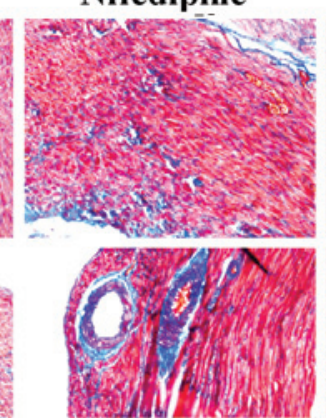

$\mathrm{D}$
OW1-H

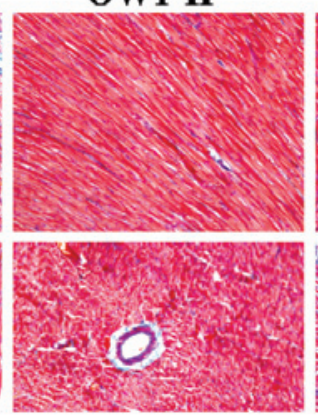

OW1-L

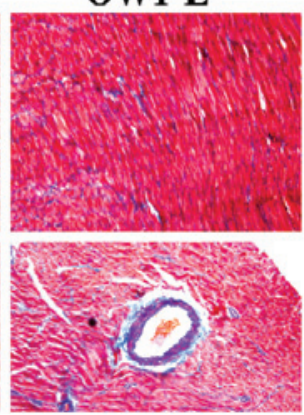

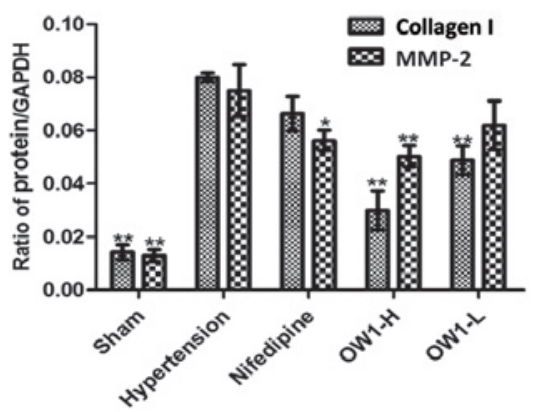

Figure 5. OW1 inhibits cardiac remodeling in 2K1C hypertensive rats in vivo. (A) Myocardial tissue and (B) blood vessels were stained with Masson's trichrome (magnification, x400). (C) The protein expression levels of collagen I and MMP-2 were determined by western blot analysis. (D) Collagen I and MMP-2 expression levels, determined from three different sets of western blots. All values are means \pm standard error of the mean $(\mathrm{n}=3)$. ${ }^{* *} \mathrm{P}<0.01$, ${ }^{*} \mathrm{P}<0.05 \mathrm{vs}$. hypertension group. OW1, an imperatorin derivative; OW1-H, high dose ( $80 \mathrm{mg} / \mathrm{kg} / \mathrm{day})$ of OW1; OW1-L, low dose (40 mg/kg/day) of OW1; 2K1C, two-kidney one-clip; MMP, matrix metalloproteinase; GAPDH, glyceraldehyde-3-phosphate dehydrogenase.

\section{Discussion}

The $2 \mathrm{~K} 1 \mathrm{C}$ renovascular hypertensive rat is a widely used model of chronic hypertension; it resembles human renovascular hypertension, with activation of the RAAS and increased
Ang II levels, which promotes vascular hypertrophy and cardiac remodeling (22). The initial cardiac hypertrophic response to hypertension in the $2 \mathrm{~K} 1 \mathrm{C}$ model involves an increase in collagen deposition, which may be due to imbalanced MMP-2 and tissue inhibitor of metalloproteinase-4 levels, resulting in increased 


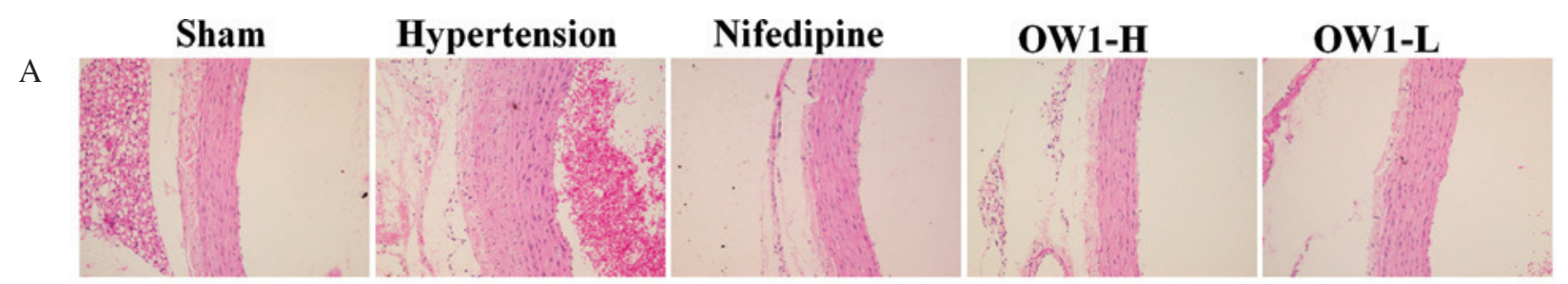

B

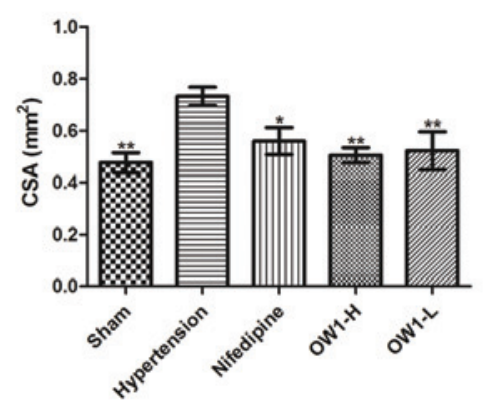

$\mathrm{C}$

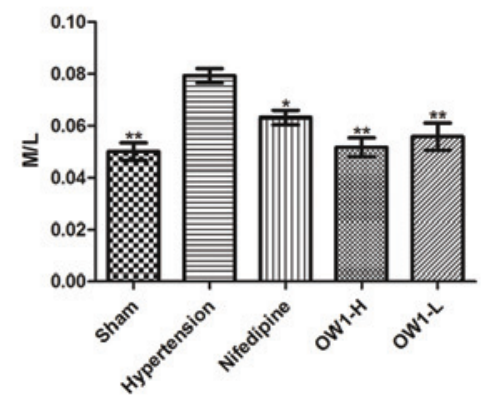

Figure 6. OW1 inhibits vascular remodeling in 2K1C hypertensive rats in vivo. (A) Thoracic arteries were stained with hematoxylin and eosin (magnification, $\mathrm{x} 400)$. Bar graphs showing (B) aortic medial cross-sectional area (CSA) and (C) aortic artery media to lumen (M/L) ratios. All values are means \pm standard error of the mean $(\mathrm{n}=8) .{ }^{* *} \mathrm{P}<0.01,{ }^{*} \mathrm{P}<0.05$ vs. hypertension group. OW1, an imperatorin derivative; OW1-H, high dose (80 mg/kg/day) of OW1; OW1-L, low dose (40 mg/kg/day) of OW1; 2K1C, two-kidney one-clip.

gelatinolytic activity, and elevated TGF- $\beta$ and ROS levels that persist until later phases of hypertension (23).

In the present study, the blood pressure in the hypertensive rats was markedly reduced by OW1, and the antihypertensive effects of high-dose OW1 were comparable with those of nifedipine. The effect of OW1 on VSMC proliferation was investigated in vitro. OW1 $(5,10$ and $20 \mu \mathrm{M})$ inhibited Ang II-induced VSMC proliferation and the phenotypic modulation of VSMCs induced by Ang II, indicated by the finding that OW1 increased $\alpha$-SMA levels. Moreover, OW1 inhibited VSMC proliferation in $2 \mathrm{~K} 1 \mathrm{C}$ renovascular hypertensive rats in vivo. The histology results illustrated that OW1 decreased the number of VSMCs in the vessel wall and reduced vascular hypertrophy, with low arterial CSA and M/L ratios in the OW1 groups. A particularly notable observation in the present study was that OW1 reduced cardiac remodeling. It has been reported that cardiac weight increases from 15 to 75 days after arterial clipping in $2 \mathrm{~K} 1 \mathrm{C}$ hypertensive rats (23). Increased cardiac weight is associated with cardiomyocyte hypertrophy and increased collagen deposition in hypertensive animals (8). In the present study, OW1 decreased the cardiac weight and fibrosis in the myocardium or around the microvasculature was barely visible in the OW1 groups. Western blotting results also documented that OW1 decreased collagen I expression in the cardiac muscle compared with that in the hypertension group. Activation of the RAAS may induce cardiac alterations, with increased Ang II formation causing TGF- $\beta$ activation resulting in cardiac hypertrophy and fibrosis $(24,25)$. Ang II, in addition to constricting blood vessels and stimulating the adrenal cortex to secrete ALD, increase blood volume and elevate blood pressure, also has a role in cardiovascular remodeling, heart failure, arterial atherosclerosis and glomerular sclerosis (26-28). Ang II exerts hypertrophic effects via the formation of ROS $(29,30)$, which increase TGF- $\beta$ levels, a requisite for the development of Ang II-induced cardiac hypertrophy (31). TGF- $\beta$ indirectly activates MMPs by involvement in the transition of fibroblasts to myofibroblasts, which produce MMPs (1). MMPs contribute to the progression of cardiac hypertrophy to heart failure (32-35); they degrade fibrillar collagen and contribute to cardiac dysfunction and remodeling of the left ventricle in hypertension $(2,36)$. Notably, the overexpression of MMP-2 has been found to induce profound ventricular remodeling and systolic dysfunction in the absence of superimposed injury (37), which may be a result of MMP-2 acting on intracellular targets and potentially impairing myocardial contractility (38). Increased cardiac levels of MMP-2 have been found to be co-localized with gelatinolytic activity in the heart tissue during all phases of $2 \mathrm{~K} 1 \mathrm{C}$-induced hypertension (23). The results of the present study showed that OW1 decreased the TGF- $\beta$ levels in plasma, and the levels of Ang II and MMP-2 in heart tissue, which clarify the inhibitory effect of OW1 on cardiovascular remodeling.

The results of the present study suggested that OW1 had antihypertensive and inhibitory effects on vascular and cardiovascular remodeling. Therefore, OW1 may reduce the risk of hypertension-induced cardiovascular diseases, which has potential clinical implications.

\section{Acknowledgements}

The present study was supported by the National Natural Science Foundation of China (grant nos. 81230079, 81227802 and 81202494).

\section{References}

1. Berk BC, Fujiwara K and Lehoux S: ECM remodeling in hypertensive heart disease. J Clin Invest 117: 568-575, 2007.

2. Opie LH, Commerford PJ, Gersh BJ and Pfeffer MA: Controversies in ventricular remodelling. Lancet 367: 356-367, 2006.

3. Arribas SM, Hinek A and González MC: Elastic fibres and vascular structure in hypertension. Pharmacol Ther 111: 771-791, 2006. 
4. Humphrey JD: Mechanisms of arterial remodeling in hypertension: Coupled roles of wall shear and intramural stress. Hypertension 52: 195-200, 2008.

5. Rossi MA: Pathologic fibrosis and connective tissue matrix in left ventricular hypertrophy due to chronic arterial hypertension in humans. J Hypertens 16: 1031-1041, 1998.

6. Rossi MA and Peres LC: Effect of captopril on the prevention and regression of myocardial cell hypertrophy and interstitial fibrosis in pressure overload cardiac hypertrophy. Am Heart J 124 700-709, 1992.

7. Rosenkranz S: TGF-beta1 and angiotensin networking in cardiac remodeling. Cardiovasc Res 63: 423-432, 2004.

8. Rizzi E, Castro MM, Ceron CS, Neto-Neves EM, Prado CM, Rossi MA, Tanus-Santos JE and Gerlach RF: Tempol inhibits TGF- $\beta$ and MMPs upregulation and prevents cardiac hypertensive changes. Int J Cardiol 165: 165-173, 2013.

9. Sadoshima J and Izumo S: Molecular characterization of angiotensin II-induced hypertrophy of cardiac myocytes and hyperplasia of cardiac fibroblasts. Critical role of the AT1 receptor subtype. Circ Res 73: 413-423, 1993.

10. Zhao W, Zhao T, Chen Y, Ahokas RA and Sun Y: Oxidative stress mediates cardiac fibrosis by enhancing transforming growth factor-betal in hypertensive rats. Mol Cell Biochem 317: 43-50, 2008.

11. Bergman MR, Teerlink JR, Mahimkar R, Li L, Zhu BQ, Nguyen A, Dahi S, Karliner JS and Lovett DH: Cardiac matrix metalloproteinase-2 expression independently induces marked ventricular remodeling and systolic dysfunction. Am J Physiol Heart Circ Physiol 292: H1847-H1860, 2007.

12. Sawicki G, Leon H, Sawicka J, Sariahmetoglu M, Schulze CJ, Scott PG, Szczesna-Cordary D and Schulz R: Degradation of myosin light chain in isolated rat hearts subjected to ischemia-reperfusion injury: A new intracellular target for matrix metalloproteinase-2. Circulation 112: 544-552, 2005

13. Wang W, Schulze CJ, Suarez-Pinzon WL, Sariahmetoglu M, Schulze CJ, Scott PG, Szczesna-Cordary D and Schulz R: Intracellular action of matrix metalloproteinase-2 accounts for acute myocardial ischemia and reperfusion injury. Circulation 106 : $1543-1549,2002$

14. Zhou N, Wang T, Song J, He H, He J and He L: Antihypertensive and vascular remodelling effects of the imperatorin derivative OW1 in renovascular hypertension rats. Clin Exp Pharmacol Physiol 41: 571-578, 2014.

15. Doggrell SA and Brown L: Rat models of hypertension, cardiac hypertrophy and failure. Cardiovasc Res 39: 89-105, 1998.

16. Committee for the Update of the Guide for the Care and Use of Laboratory Animals; National Research Council: Guide for the Care and Use of Laboratory Animals 8th edition. National Academies Press, Washington, DC, 2011.

17. Wang C, Wang T, Zhou N, Pan XY and He HZ: Design, synthesis and evaluation of 9-hydroxy-7H-furo[3,2-g]chromen-7-one derivatives as new potential vasodilatory agents. J Asian Nat Prod Res 16: 304-311, 2014

18. Gordon D, Mohai LG and Schwartz SM: Induction of polyploidy in cultures of neonatal rat aortic smooth muscle cells. Circ Res 59: 633-644, 1986

19. Marshall NJ, Goodwin CJ and Holt SJ: A critical assessment of the use of microculture tetrazolium assays to measure cell growth and function. Growth Regul 5: 69-84, 1995.

20. Maliszewska-Scislo M, Chen H, Augustyniak RA, Seth D and Rossi NF: Subfornical organ differentially modulates baroreflex function in normotensive and two-kidney, one-clip hypertensive rats. Am J Physiol Regul Integr Comp Physiol 295: R741-R750, 2008.

21. Fritz $M$ and Rinaldi G: Influence of nitric oxide-mediated vasodilation on the blood pressure measured with the tail-cuff method in the rat. J Biomed Sci 14: 757-765, 2007.

22. Ceron CS, Rizzi E, Guimaraes DA, Martins-Oliveira A, Cau SB, Ramos J, Gerlach RF and Tanus-Santos JE: Time course involvement of matrix metalloproteinases in the vascular alterations of renovascular hypertension. Matrix Biol 31: 261-270, 2012.
23. Rizzi E, Ceron CS, Guimaraes DA, Prado CM, Rossi MA, Gerlach RF and Tanus-Santos JE: Temporal changes in cardiac matrix metalloproteinase activity, oxidative stress, and TGF- $\beta$ in renovascular hypertension-induced cardiac hypertrophy. Exp Mol Pathol 94: 1-9, 2013.

24. Schlumberger W, Thie M, Rauterberg J and Robenek H: Collagen synthesis in cultured aortic smooth muscle cells. Modulation by collagen lattice culture, transforming growth factor-beta 1 and epidermal growth factor. Arterioscler Thromb 11: 1660-1666, 1991.

25. Zhang LF, Ding WH, Shi LB, Li K, Haom YJ, Ke YN and Tang ZS: Effects of exogenous urotensin II on vascular remodelling after balloon injury. Clin Exp Pharmacol Physiol 37: 477-481, 2010

26. Browatzki M, Larsen D, Pfeiffer CA, Gehrke SG, Schmidt J, Kranzhofer A, Katus HA and Kranzhofer R: Angiotensin II stimulates matrix metalloproteinase secretion in human vascular smooth muscle cells via nuclear factor-kappaB and activator protein 1 in a redox-sensitive manner. J Vasc Res 42: 415-423, 2005.

27. Kranzhöfer R, Schmidt J, Pfeiffer CA, Hagl S, Libby P and Kübler W: Angiotensin induces inflammatory activation of human vascular smooth muscle cells. Arterioscler Thromb Vasc Biol 19: $1623-1629,1999$

28. van Leeuwen RT, Kol A, Andreotti F, Kluft C, Maseri A and Sperti G: Angiotensin II increases plasminogen activator inhibitor type 1 and tissue-type plasminogen activator messenger RNA in cultured rat aortic smooth muscle cells. Circulation 90: 362-368, 1994.

29. Baker KM and Aceto JF: Angiotensin II stimulation of protein synthesis and cell growth in chick heart cells. Am J Physiol 259: H610-H618, 1990.

30. Sorescu D and Griendling KK: Reactive oxygen species, mitochondria and $\mathrm{NAD}(\mathrm{P}) \mathrm{H}$ oxidases in the development and progression of heart failure. Congest Heart Fail 8: 132-140, 2002.

31. Schultz Jel J, Witt SA, Glascock BJ, Nieman ML, Reiser PJ, Nix SL, Kimball TR and Doetschman T: TGF-betal mediates the hypertrophic cardiomyocyte growth induced by angiotensin II. J Clin Invest 109: 787-796, 2002 .

32. Fert-Bober J, Leon H, Sawicka J, Basran RS, Devon RM, Schulz R and Sawicki G: Inhibiting matrix metalloproteinase-2 reduces protein release into coronary effluent from isolated rat hearts during ischemia-reperfusion. Basic Res Cardiol 103: 431-443, 2008.

33. Wang GY, Bergman MR, Nguyen AP, Turcato S, Swigart PM, Rodrigo MC, Simpson PC, Karliner JS, Lovett DH and Baker AJ: Cardiac transgenic matrix metalloproteinase-2 expression directly induces impaired contractility. Cardiovasc Res 69: 688-696, 2006.

34. Iwanaga Y, Aoyama T, Kihara Y, Onozawa Y, Yoneda T and Sasayama S: Excessive activation of matrix metalloproteinases coincides with left ventricular remodeling during transition from hypertrophy to heart failure in hypertensive rats. J Am Coll Cardiol 39: 1384-1391, 2002

35. Spinale FG: Myocardial matrix remodeling and the matrix metalloproteinases: Influence on cardiac form and function. Physiol Rev 87: 1285-1342, 2007

36. Takenaka H, Kihara Y, Iwanaga Y, Onozawa Y, Toyokuni S and Kita T: Angiotensin II, oxidative stress and extracellular matrix degradation during transition to LV failure in rats with hypertension. J Mol Cell Cardiol 41: 989-997, 2006.

37. Castro MM, Rizzi E, Figueiredo-Lopes L, Fernandes K, Bendhack LM, Pitol DL, Gerlach RF and Tanus-Santos JE: Metalloproteinase inhibition ameliorates hypertension and prevents vascular dysfunction and remodeling in renovascular hypertensive rats. Atherosclerosis 198: 320-331, 2008.

38. Mujumdar VS, Smiley LM and Tyagi SC: Activation of matrix metalloproteinase dilates and decreases cardiac tensile strength. Int J Cardiol 79: 277-286, 2001. 